\title{
Tooth decay: Is today's grazing culture contributing to the high prevalence of tooth decay?
}

\author{
Despite a greater proportion of dentate adults in the UK engaging in good \\ oral health behaviours than ever before, ${ }^{1}$ dental caries continues to be one of
} the most common dental diseases in the UK. ${ }^{2}$ Only one in ten UK adults meet the criteria of having excellent oral health, ${ }^{*}$ with approximately a third having obvious dental caries. ${ }^{1}$

\begin{abstract}
So why does tooth decay continue to be a problem?

The gap between what and when we eat or drink and the measures we take to protect our teeth may be a contributing factor to this high prevalence of tooth decay. New data presented in the recent Eat, Drink, Think report published by the Wrigley Oral Healthcare Programme, presents evidence that suggests there has been a shift in eating habits of UK adults and identifies an increase in snacking between meals - a trend known as 'grazing. The report revealed that most respondents $(83 \%)$ consume at least one snack between meals and almost half (48\%) enjoy two snacks or more per day. ${ }^{3}$ The data supports the 2010 report on oral health by the European Commission, which found the frequency of snacking in the UK is above the European average, with UK respondents reporting an average of 6.7 eating or drinking occasions per day compared with a European average of 5.4 occasions. ${ }^{4}$

Although brushing twice daily with
\end{abstract}

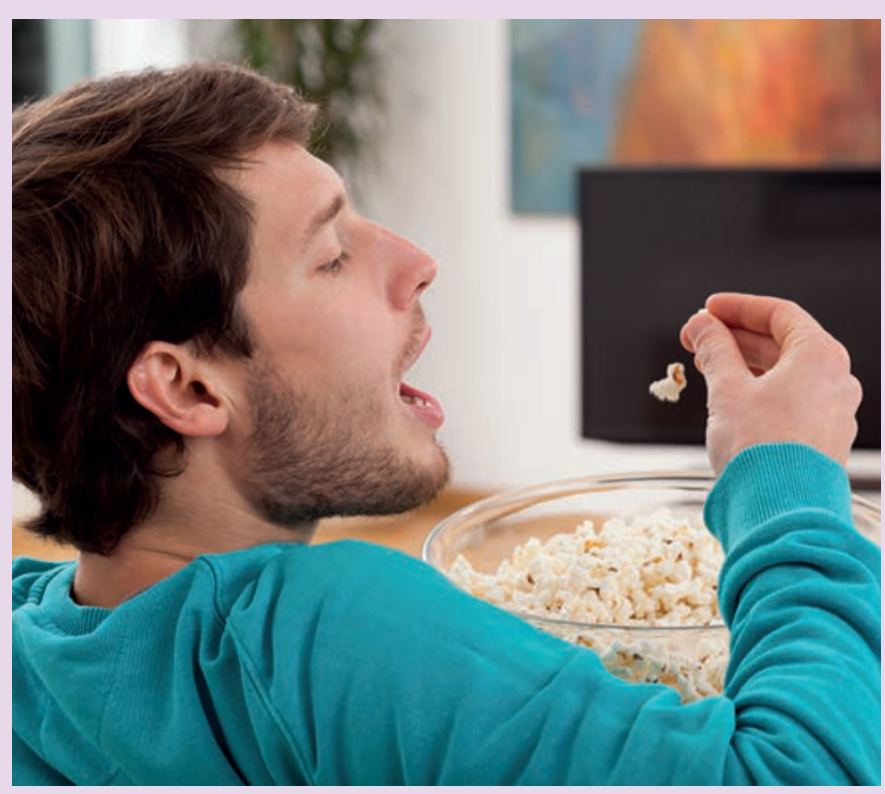

fluoridated toothpaste is still the most important method for preventing caries, the amount and frequency of intake of cariogenic foods and drinks have an influence on our oral health. $^{1,5}$

Data presented in Eat, Drink, Think shows that a significant number of respondents reported no oral health intervention after $56 \%$ of morning snacks and $60 \%$ of afternoon snacks. ${ }^{3}$ Reflecting on our eating and drinking habits, the report suggests that the current oral health guidelines - based on the assumption we consume three meals a day - may no longer be sufficient to maintain good oral health.

Research suggests that three quarters of UK adults brush their teeth at least twice a day and over two-thirds visit a dentist at least once a year. ${ }^{3}$ With such large numbers already following official advice, a simple low-cost preventative addition to current guidelines for example, recommending appropriate oral health interventions after eating and drinking such as chewing sugarfree gum - would build on existing oral hygiene behaviours and help support better oral health in between brushing.

As proven by independent clinical research, chewing sugarfree gum for 20 minutes after eating and drinking effectively stimulates saliva and helps to neutralise plaque acid that can cause tooth decay. ${ }^{6}$ In addition to the oral health benefits of chewing sugarfree gum, there is also the potential for significant economic benefits. Research published in this Journal showed that if all UK 12-year-olds were to chew one additional piece of sugarfree gum per day, the NHS could save up to $£ 2.8$ million in tooth decay costs per year. ${ }^{7}$ This cost saving rises to a potential $£ 8.2$ million if three pieces of sugarfree gum were to be chewed per day, ${ }^{7}$ the equivalent of 364,000 dental check-ups. ${ }^{8}$

Eat, Drink, Think found that two thirds of respondents were not fully aware of the oral health benefits of chewing sugarfree gum, but $42 \%$ said they'd be more likely to chew sugarfree gum after being told of these benefits. $^{3}$

To view the complete findings of Eat, Drink, Think visit wrigleyoralhealthcare.co.uk.

*To identify the proportion of dentate adults who met these criteria, a composite measure was created. This measure of excellent oral health prospects comprised people who met all of the following criteria: 21 or more natural teeth; 18 or more sound and untreated teeth and roots; no decay detected at any site; no periodontal pocketing of $4 \mathrm{~mm}$ or more and no loss of attachment of $4 \mathrm{~mm}$ or more; no calculus or bleeding.

1. Adult Dental Health Survey 2009 - England, Wales, Northern Ireland, 2009. Available at: http://content.digital.nhs.uk/pubs/dentalsurveyfullreport09 (accessed July 2017).

2. Steele J. An Independent Review of NHS Dental Services in England. 2009. Available at: http://www. sigwales.or g/wp-content/uploads/dh_101180.pdf (accessed July 2017).

3. Kantar TNS' online omnibus survey (Onlinebus) of 2,743 UK adults. Conducted April 2017.

4. European Commission. Special Eurobarometer 330. Oral Health. Brussels: European Commission, 2010. Available at: http://ec.europa.eu/public_opinion/ archives/ebs/ebs_330_en.pdf (accessed September 2017).

5. Public Health England (Department of Health). Delivering better oral health: an evidence-based toolkit for prevention. March 2017. Available at: https://www. gov.uk/government/publications/delivering-betteroral-health-an-evidence-based-toolkit-for-prevention (accessed September 2017).

6. De Almeida P D, Gregio A M, Machado M A et al. Saliva composition and functions: a comprehensive review. J Contemp Dent Pract 2008; 9: 72-80.

7. Claxton L, Taylor M, Kay E. The economic benefits to the NHS of increased use of sugarfree gum use in the UK. Br Dent J 2016; 220: 121-127.

8. 1 Unit of dental activity $=£ 22.50 .8200,000 / 22.50=$ 364,000 . 